\section{Finding the cause of that facial swelling}

\section{Samantha Woods}

Facial swelling in dogs and cats can develop as a fairly benign condition or may become life threatening, requiring emergency treatment. There are a number of different causes of swelling that should be investigated to allow appropriate treatment decisions to be made.

Common causes of facial swelling include:

\section{- Allergy \\ - Abscess \\ - Bite/trauma \\ - Dental disease \\ - Tumours.}

Less common causes include:

- Caval syndrome

- Craniomandibular osteopathy

- Toxin ingestion (e.g. paracetamol in cats).

In some cases, swelling may be suspected but, on more detailed physical examination, asymmetry of the face associated with muscle wastage may be identified (e.g. trigeminal neuropathy).

The signalment, physical examination and owner consultation can provide relevant information that will help ascertain the most likely cause. Is the patient young or old? Does the patient go outside (cats)? Is there a history of trauma? Is the swelling acute or chronic in onset? Where is the swelling - is it localized or generalized and over what anatomical area? Is there a wound? Is the swollen area solid on palpation, or does the swelling feel fluid-filled? Is the swelling painful or itchy? Is there any other sign that the patient is systemically affected reduced appetite, lethargy, pain on eating?

Allergies are a common cause of swelling in both dogs and cats and finding a cause can be extremely difficult. Initial treatment with antihistamines or steroids may be warranted with further, more detailed investigations including diet trials and allergy tests planned if the condition continues. In severe cases of allergy, swelling of the respiratory system may occur and these cases must be treated as emergencies.

Abscesses are very common in dogs, cats and rabbits. They can be due to bites, trauma, puncture wounds and dental disease. Patients often present with pain and pyrexia as well as an asymmetric swelling. Cellulitis and oedema may also develop. Radiographs, ultrasonography and computed tomography are used to identify the cause. Treatment includes flushing of the abscess, marsupialization, appropriate antibiotic therapy and management of the underlying disease. Retrobulbar abscesses may also occur, leading to exophthalmos and facial swelling.

Tumours of the head are fairly common in dogs and cats. They often present as localized lesions and may or may not ulcerate. Incisional biopsy assists with treatment planning and investigation of local lymph nodes for metastasis is recommended in all cases where a tumour of the head or neck is suspected.

This talk will discuss the causes of facial swelling, provide a logical investigative path to identify the cause and briefly mention treatment options where appropriate. Case-based material will be used to illustrate these concepts wherever possible.

\section{KEY LEARNING OBJECTIVES}

- Recognize that there are many different causes of facial swelling, some more commonly identified than others

- Signalment, history and physical examination can provide useful information regarding the potential cause of the condition and detailed questioning of the owner is advised in all cases

- The diagnostic work-up should consider all the potential causes and aim to rule them in or out in a logical step-by-step manner

\section{MULTIPLE CHOICE QUESTIONS}

1. What is the most common cause of retrobulbar swelling in dogs?

(A) Inflammatory/infectious

(B) Neoplastic

(C) Trauma

(D) Both $A$ and $B$

2. You are presented with a cat with generalized facial swelling, chocolate brown mucous membranes, tachycardia and dyspnoea. What is the most likely cause?

(A) Ethylene glycol ingestion

(B) Organophosphate ingestion

(C) Paracetamol ingestion

(D) Warfarin ingestion

3. You are evaluating a patient that is presented with facial swelling. On physical examination you discover asymmetry in the facial muscles with atrophy on the left side, no blink response on the left, and no nasal sensation on the left. Which nerve is most likely to be affected?
(A) $\mathrm{CN}$ III
(B) CN IV
(C) $\mathrm{CN} \mathrm{V}$
(D) CN VII 